\title{
PRAGAS E INIMIGOS NATURAIS NA SOJA E NO MILHO CULTIVADOS EM SISTEMAS DIVERSIFICADOS
}

\author{
Francisco Jorge Cividanes ${ }^{1,3 *}$; Fábio Takeshi Yamamoto² \\ ${ }^{1}$ Depto. de Fitossanidade - FCAVIUNESP, Via de Acesso Prof. Paulo Donato Castellane, s/n - CEP: 14884-900 - \\ Jaboticabal, SP. \\ ${ }^{2}$ Bayer S.A. - R. Domingos Jorge, 1000 - CEP: 04779-900 - São Paulo, SP. \\ ${ }^{3}$ Bolsista CNPq. \\ *Autor correspondente <fjcivida@fcav.unesp.br>
}

\begin{abstract}
RESUMO: A consorciação de culturas e o plantio direto podem influenciar a dinâmica populacional de insetos, aumentando ou diminuindo a densidade populacional de pragas e de inimigos naturais. Deste modo, avaliaramse os efeitos de sistemas de plantio direto e da consorciação soja [Glycine max (L.) Merrill] e milho (Zea mays L.) sobre as populações de insetos-pragas e inimigos naturais. Os tratamentos constituíram um fatorial 3x2 (monocultura de soja, monocultura de milho, consorciação soja-milho em plantio direto e plantio convencional). Os insetos foram amostrados pelo método do pano, rede entomológica e procura visual. No milho, a ocorrência de insetos-pragas da parte aérea e insetos predadores não foi influenciada pelo manejo do solo. Lagartas de Helicoverpa zea ocorreram em maior número $(5,1)$ no milho em consorciação. Na soja, adultos de Anticarsia gemmatalis e do predador Cycloneda sanguinea foram mais abundantes em plantio direto, respectivamente, 0,4 e 2,4 insetos. Observou-se maior número do sirfídeo Toxomerus sp. em plantio convencional $(1,3)$. Nas condições de consorciação, verificou-se baixo número de Diabrotica gracilenta $(11,5)$ na soja, enquanto que Maecolaspis sp. $(5,0)$ e Megascelis sp. $(17,0)$ e os predadores C. sanguinea $(2,3)$, Doru sp. $(0,8)$, Geocoris sp. $(1,5)$ e Toxomerus sp. $(1,4)$ ocorreram em alta densidade.

Palavras-chave: Glycine max, Zea mays, dinâmica populacional de insetos, plantio direto, consorciação de culturas
\end{abstract}

\section{PESTS AND THEIR NATURAL ENEMIES ON SOYBEAN AND CORN GROWN IN DIVERSIFIED SYSTEMS}

\begin{abstract}
Intercropping and no-tillage systems can affect the population dynamics of insects, increasing or decreasing the population density of pests and natural enemies. The objective of this study was to evaluate the effects of no-tillage and strip intercropping of soybean [Glycine max (L.) Merrill] and corn (Zea mays L.) on the abundance of insect pests and their natural enemies. Plots were arranged in a randomized complete block design in a $3 \times 2$ factorial experiment (soybean monoculture, corn monoculture, strip intercropping of soybean-corn under no-tillage and conventional tillage). Insects were sampled using sweep net, ground cloth and by visual search. For corn, the occurrence of insect pests and predators was not affected by soil management practices. Larvae of Helicoverpa zea occurred in higher number (5.1) for corn under strip intercropping. For soybean, adults of Anticarsia gemmatalis and of the predator Cycloneda sanguinea were more abundant for the no-tillage plots, respectively, 0.4 and 2.4 insects. A higher number of the syrphid Toxomerus sp. was observed for the conventional tillage plots (1.3). Under strip intercropping conditions, low numbers of Diabrotica gracilenta (11.5) were observed, while Maecolaspis sp. (5.0) and Megascelis sp. (17.0), and the predators C. sanguinea (2.3), Doru sp. (0.8), Geocoris sp. (1.5) and Toxomerus sp. (1.4) occurred in high densities.
\end{abstract}

Key words: Glycine max, Zea mays, insect population dynamics, no-tillage, intercropping

\section{INTRODUÇÃO}

O controle biológico natural é uma alternativa para a diminuição ou eliminação do uso de inseticidas no controle de pragas (Altieri, 1989). A ocorrência de inimigos naturais pode ser aumentada pela adoção de sistemas conservacionistas de manejo do solo (Stinner \& House, 1990) e pela consorciação de culturas, sendo esta prática importante para aumentar a diversidade desses insetos (Altieri et al., 1990).

No Brasil, sistemas de plantio direto causaram redução na infestação de várias pragas nas culturas de trigo, algodão e feijão (Bianco, 1984). Na soja, o plantio direto tem beneficiado a ocorrência dos principais parasitóides de ovos dos percevejos Nezara viridula e Piezodorus guildinii (Moreira \& Becker, 1986) e os fungos causadores de doenças em lagartas (Sosa-Gómez \& Moscardi, 1994). Com relação à consorciação soja-milho, Milanez (1984) encontrou menor incidência de Anticarsia gemmatalis, $N$. viridula e Cerotoma sp. em comparação ao monocultivo dessa leguminosa.

Em outros países, sistemas de plantio direto da soja contribuíram para aumentar o complexo de inimigos naturais (Ferguson et al., 1984) e diminuir a ocorrência de várias espécies fitófagas (Hammond \& Stinner, 1987). No milho sob este mesmo sistema de 
plantio, predadores constituíram o principal fator na redução dos danos causados por Agrotis ipsilon (Brust et al., 1986).

Vários estudos têm demonstrado a abundância de insetos predadores nas culturas da soja e do milho (Hsin et al., 1979; Bechinski \& Pedigo, 1981), afetando a dinâmica populacional de pragas (Frank, 1971; Best \& Beegle, 1977; Fuller, 1988). Como o plantio direto e a consorciação de culturas podem influir na dinâmica populacional de pragas e de inimigos naturais (Risch et al., 1983), o objetivo deste estudo foi avaliar o efeito de sistemas de plantio direto e convencional e da consorciação soja-milho sobre pragas e insetos predadores.

\section{MATERIAL E MÉTODOS}

O estudo foi conduzido em uma área de 1,6 ha em Jaboticabal - SP, (2115'22" S; 48¹8'58” W; 595 m) no período de outubro de 1996 a abril de 1997. A área experimental foi cultivada com soja na safra 1995-96 utilizando-se o método convencional, seguido do plantio direto de sorgo. Depois da colheita do sorgo, permitiuse a rebrota desta gramínea, a qual foi seca com herbicida glyphosate e a palha cortada com triturador. As parcelas de plantio convencional foram aradas e gradeadas. A semeadura foi efetuada em 28/11/96 com o cultivar BR-16 de soja e o híbrido Pionner 3041 de milho, sendo em seguida aplicado herbicida.

Os tratamentos foram esquematizados em fatorial $3 \times 2$, constituindo seis tratamentos, monocultura de soja, monocultura de milho e consorciação soja-milho e cada um destes em plantio direto e plantio convencional. Utilizou-se o delineamento em blocos casualizados em quatro repetições. As parcelas ficaram dispostas em linha e separadas $10 \mathrm{~m}$ entre si. As parcelas com soja apresentaram 40 linhas de plantas espaçadas $0,5 \mathrm{~m}$ entre si e dimensões de $16 \times 20 \mathrm{~m}$. As parcelas de milho foram constituídas por 20 linhas de plantas espaçadas $0,90 \mathrm{~m}$ e dimensões de $16 \times 18 \mathrm{~m}$. As parcelas com culturas consorciadas foram formadas por três faixas de milho com cinco linhas de planta cada uma, alternadas com duas faixas de 10 linhas de soja com dimensões de $16 \times 2,35 \mathrm{~m}$. Os dados foram submetidos à análise de variância e as médias comparadas pelo teste de Tukey a $5 \%$.

$\mathrm{Na}$ soja, os inimigos naturais e as pragas da parte aérea foram amostrados em nove épocas, utilizando-se o método do pano e a rede entomológica (Tonhasca Jr., 1993). No início do desenvolvimento das plantas, os insetos foram amostrados por procura visual em $6 \mathrm{~m}$ por parcela, até que as mesmas atingissem a altura de $20 \mathrm{~cm}$, quando passaram a ser amostradas pelo método do pano em três pontos escolhidos ao acaso dentro da parcela. A amostragem com rede entomológica (20 redadas por parcela) foi iniciada na mesma época da procura visual.
No milho, as pragas da parte aérea foram amostradas através de procura visual, escolhendo-se ao acaso cinco pontos por parcela, cada ponto constituído por $2 \mathrm{~m}$ de plantas. Para a avaliação da ocorrência da lagarta do cartucho, Spodoptera frugiperda, considerouse o número de plantas que apresentavam-se danificadas pela praga. Esta amostragem foi realizada semanalmente desde a emergência do milho até o estádio três (12 folhas emergidas) (Fornasieri Filho, 1992), totalizando cinco épocas de amostragem. O percevejo Leptoglossus zonatus teve sua amostragem iniciada com o surgimento das espigas, sendo realizadas cinco amostragens por procura visual nas espigas presentes nas plantas dos pontos escolhidos na parcela. A amostragem da lagarta da espiga, Helicoverpa zea, consistiu na retirada, ao acaso, de dez espigas por parcela, em três épocas com intervalo de cerca de 10 dias, e iniciada no momento em que se constatou a presença de lagartas atacando as espigas. Os insetos predadores foram amostrados por meio de procura visual, totalizando sete épocas de amostragem. Neste procedimento, foram escolhidos ao acaso cinco pontos por parcela, cada um dos quais constituídos por $2 \mathrm{~m}$ de plantas de milho.

\section{RESULTADOS E DISCUSSÃO}

No milho consorciado com soja verificou-se maior número de espigas atacadas por $H$. zea em relação à monocultura do milho (Tabela 1). Picanço et al. (1996) também relataram um incremento no ataque dessa espécie de praga aos frutos do tomateiro conduzido em consorciação com milho. Segundo Ramaswamy (1988), insetos polífagos como $H$. zea apresentam oviposição não seletiva. Tais espécies de insetos caracterizam-se por localizarem a planta hospedeira pela textura superficial, odores e quimioreceptores de contato. Assim, a maior ocorrência de $H$. zea no milho consorciado com soja no presente estudo deve-se, possivelmente, a alguma interferência causada por esse tipo de cultivo no mecanismo de $H$. zea localizar a planta hospedeira. Ainda no sistema de cultivo citado anteriormente, $S$. frugiperda e $L$. zonatus apresentaram-se igualmente abundantes no milho consorciado e na monocultura dessa gramínea (Tabela 1). Os resultados obtidos para $S$. frugiperda são similares àqueles encontrados por Castro et al. (1994), que não observaram diferença na ocorrência dessa praga em milho como monocultura ou em consorciação com ervilha. Nos sistemas de plantio direto e convencional do milho não se constatou diferença na ocorrência de $S$. frugiperda, $H$. zea e $L$. zonatus (Tabela 1). Stinner et al. (1988), estudando comunidades de artrópodes em milho cultivado em áreas com 20 anos de práticas de plantio convencional e direto, também observaram que a densidade populacional de várias pragas não foi influenciada pelo sistema de manejo do solo, destacando-se entre elas Ostrinia nubilalis e Diabrotica longicornis. 
Com exceção de adultos de $A$. gemmatalis, que apresentaram maior ocorrência na soja sob plantio direto quando comparada à de plantio convencional, as demais pragas não foram influenciadas pelo sistema de plantio (Tabelas 2 e 3). A maior densidade de adultos de $A$. gemmatalis em plantio direto pode estar relacionada com a presença de restos vegetais sobre o solo, o que possibilitou a emissão de radiação infra-vermelha dentro de uma faixa mais atrativa para os referidos adultos do que a radiação infra-vermelha emitida nas condições de plantio convencional. Mariposas são atraídas pela radiação infra-vermelha, cuja intensidade e comprimento de onda dependem do material emissor (Silveira Neto et al., 1976).

$\mathrm{Na}$ soja consorciada com milho, apenas crisomelídeos mostraram-se influenciados por esse tipo de cultivo. Diabrotica gracilenta ocorreu em menor número na soja consorciada com milho (Tabela 2), o oposto sendo observado para Megascelis sp. e Maecolaspis sp. (Tabela 3). Ellsbury et al. (1999) também constataram diferenças entre a abundância de espécies de crisomelídeos em sistemas de consorciação sojamilho. No presente trabalho, não foram determinadas as causas que possibilitaram as espécies de crisomelídeos apresentarem densidades populacionais contrastantes na soja consorciada com milho. No entanto, a variação nas populações de artrópodes depende da densidade de plantio, culturas envolvidas, adaptabilidade do predador à cultura, densidade da presa, especificidade do predador, disponibilidade de outras fontes de alimento como pólen, umidade do solo e microclima das culturas (Stinner \& House, 1990; Booij \& Noorlander, 1992; Cárcamo \& Spence, 1994; Clark et al., 1997; French et al., 1998), evidenciando a necessidade de estudos que considerem a complexidade da interação planta-inseto em sistemas diversificados, para possibilitarem maior entendimento de sua dinâmica populacional.

Tesourinhas da espécie Doru luteipes têm se mostrado importantes predadores de pragas associadas ao milho, principalmente S. frugiperda e H. zea (Cruz \& Oliveira, 1997). Com relação aos insetos predadores encontrados no milho no presente estudo, Doru sp. foi a espécie observada em maior número (Tabela 1 ). No entanto, a ocorrência desse predador não foi influenciada pelo manejo do solo e consorciação com soja, o mesmo verificando-se com C. sanguinea. Tonhasca Jr. \& Stinner (1991) obtiveram resultados semelhantes para a abundância de artrópodes predadores em milho sob as mesmas condições de cultivo do presente estudo.

Entre os insetos predadores capturados na soja, observou-se maior número de $C$. sanguinea no plantio direto que no convencional. Situação oposta ocorreu com o sirfídeo Toxomerus sp., enquanto Doru sp. e Geocoris sp. tiveram ocorrência semelhante nesses dois sistemas de plantio (Tabelas 2 e 3 ). $O$ fato de lagartas de $A$. gemmatalis não terem ocorrido em maior número na soja sob plantio direto, apesar de adultos dessa praga terem sido mais abundantes naquelas condições de manejo do solo (Tabela 3 ), talvez seja indicativo da predação efetuada por $C$. sanguinea sobre $A$. gemmatalis, uma vez que coccinelídeos alimentam-se de ínstares iniciais e ovos de lepidópteros (Bruck \& Lewis, 1998; Cottrell \& Yeargan, 1998), sendo eficientes no controle biológico de lepidópteros pragas como $\mathrm{H}$. zea e O. nubilalis (Coll \& Bottrell, 1991).

$\mathrm{Na}$ soja consorciada, as espécies de insetos predadores C. sanguinea, Doru sp., Geocoris sp. e Toxomerus sp. foram mais abundantes em relação à monocultura dessa leguminosa (Tabela 3). Tonhasca Jr. (1993) também constatou maior incidência de inimigos naturais em soja consorciada com milho. Pelos resultados do presente estudo, pode-se inferir que o sistema de consorciação soja-milho proporcionou que insetos predadores ocorressem em maior número na soja, aumentando o potencial de controle biológico natural de pragas dessa cultura. A maior abundância de insetos predadores está de acordo com a "Hipótese do Inimigo" proposta por (Root, 1973), que sugere ocorrer maior número de inimigos naturais em agroecossistemas diversificados.

Vários fatores podem explicar a maior abundância de insetos predadores na soja consorciada com milho. O microclima nas faixas de soja pode ter sido alterado pela presença do milho, proporcionando maior sombra e umidade e temperaturas mais baixas, condições que favorecem o aumento do número de

Tabela 1 - Número médio de insetos pragas e insetos predadores ( \pm erro padrão da média) amostrados por procura visual em milho. Jaboticabal, SP - 1996/97; pragas $n=5$, predadores $n=7$.

\begin{tabular}{|c|c|c|c|c|c|c|}
\hline \multirow{2}{*}{ Praga / Predador } & \multicolumn{2}{|c|}{ Manejo do Solo ${ }^{1}$} & \multicolumn{2}{|c|}{ Cultura $^{1}$} & \multirow{2}{*}{$\begin{array}{c}\text { DMS } \\
\text { Tukey 5\% }\end{array}$} & \multirow{2}{*}{$\begin{array}{c}\text { CV } \\
\%\end{array}$} \\
\hline & Direto & Convencional & Milho & Soja $x$ Milho & & \\
\hline Helicoverpa zea ${ }^{2}$ & $4,8 \pm 0,21 a^{3}$ & $4,1 \pm 0,71 a$ & $3,8 \pm 0,30 b$ & $5,1 \pm 0,57 \mathrm{a}$ & 1,17 & 23,2 \\
\hline Leptoglossus zonatus & $1,4 \pm 3,24 a$ & $1,6 \pm 2,41 a$ & $1,8 \pm 2,78 a$ & $1,2 \pm 4,18 \mathrm{a}$ & 0,88 & 52,1 \\
\hline Spodoptera frugiperda & $4,2 \pm 4,14 a$ & $3,0 \pm 3,35 a$ & $3,0 \pm 3,98 a$ & $4,2 \pm 5,03 a$ & 1,89 & 46,3 \\
\hline Cycloneda sanguinea & $1,0 \pm 0,14 \mathrm{a}$ & $0,9 \pm 0,08 a$ & $0,8 \pm 0,02 \mathrm{a}$ & $1,1 \pm 0,16 \mathrm{a}$ & 0,57 & 50,9 \\
\hline Doru sp. & $34,0 \pm 2,79 a$ & $36,7 \pm 6,56 \mathrm{a}$ & $35,2 \pm 4,38 a$ & $35,6 \pm 9,97 a$ & 4,72 & 11,8 \\
\hline
\end{tabular}

${ }^{1}$ Interações entre manejo do solo e culturas não foram significativas.

${ }^{2}$ Amostragem realizada por meio de coleta de espigas $(n=3)$.

${ }^{3}$ Médias seguidas da mesma letra na horizontal não diferem pelo teste de Tukey a $5 \%$. 
Tabela 2 - Número médio de insetos pragas e insetos predadores ( \pm erro padrão da média) amostrados pelo método do pano em soja. Jaboticabal, SP - 1996/97; $n=9$.

\begin{tabular}{|c|c|c|c|c|c|c|}
\hline \multirow{2}{*}{ Praga / Predador } & \multicolumn{2}{|c|}{ Manejo do Solo ${ }^{1}$} & \multicolumn{2}{|c|}{ Cultura $^{1}$} & \multirow{2}{*}{$\begin{array}{c}\text { DMS } \\
\text { Tukey 5\% }\end{array}$} & \multirow{2}{*}{$\begin{array}{l}\mathrm{CV} \\
\%\end{array}$} \\
\hline & Direto & Convencional & Milho & Soja $\times$ Milho & & \\
\hline $\begin{array}{l}\text { Anticarsia gemmatalis } \\
\text { (lagartas) }\end{array}$ & $3,5 \pm 0,29 a^{2}$ & $4,2 \pm 0,20 \mathrm{a}$ & $3,9 \pm 0,44 a$ & $3,5 \pm 0,25 a$ & 0,64 & 15,1 \\
\hline Diabrotica gracilenta & $4,1 \pm 0,93 \mathrm{a}$ & $3,7 \pm 0,33 a$ & $4,8 \pm 0,55 a$ & $3,0 \pm 0,31 b$ & 1,39 & 31,5 \\
\hline Piezodorus guildinii & $0,7 \pm 0,47 a$ & $0,5 \pm 0,09 a$ & $0,6 \pm 0,07 a$ & $0,7 \pm 0,39 a$ & 0,24 & 34,8 \\
\hline Cycloneda sanguinea & $0,3 \pm 0,01 \mathrm{a}$ & $0,3 \pm 0,03 a$ & $0,4 \pm 0,02 a$ & $0,2 \pm 0,01 \mathrm{a}$ & 0,28 & 92,9 \\
\hline Doru sp. & $0,5 \pm 0,01 a$ & $0,5 \pm 0,07 \mathrm{a}$ & $0,2 \pm 0,01 b$ & $0,9 \pm 0,05 a$ & 0,35 & 59,6 \\
\hline Geocoris sp. & $1,2 \pm 0,01 \mathrm{a}$ & $0,9 \pm 0,07 a$ & $0,9 \pm 0,03 a$ & $1,2 \pm 0,17 \mathrm{a}$ & 0,36 & 30,7 \\
\hline
\end{tabular}

${ }^{1}$ Interações entre manejo do solo e culturas não foram significativas.

${ }^{2}$ Médias seguidas da mesma letra na horizontal não diferem pelo teste de Tukey a 5\%.

Tabela 3 - Número médio de insetos pragas e insetos predadores ( \pm erro padrão da média) amostrados por rede entomológica em soja. Jaboticabal, SP - 1996/97; $n=9$.

\begin{tabular}{|c|c|c|c|c|c|c|}
\hline \multirow{2}{*}{ Praga / Predador } & \multicolumn{2}{|c|}{ Manejo do Solo ${ }^{1}$} & \multicolumn{2}{|c|}{ Cultura $^{1}$} & \multirow{2}{*}{$\begin{array}{c}\text { DMS } \\
\text { Tukey } 5 \%\end{array}$} & \multirow{2}{*}{$\begin{array}{l}\mathrm{CV} \\
\%\end{array}$} \\
\hline & Direto & Convencional & Milho & Soja $\times$ Milho & & \\
\hline Anticarsia gemmatalis (lagartas) & $0,1 \pm 0,05 \mathrm{a}^{2}$ & $0,1 \pm 0,04 a$ & $0,1 \pm 0,01 \mathrm{a}$ & $0,1 \pm 0,07 \mathrm{a}$ & 0,19 & 105,4 \\
\hline A. gemmatalis (adultos) & $0,4 \pm 0,09 a$ & $0,1 \pm 0,11 b$ & $0,2 \pm 0,61 \mathrm{a}$ & $0,3 \pm 1,04 \mathrm{a}$ & 0,26 & 94,8 \\
\hline Diabrotica gracilenta & $15,1 \pm 18,11 \mathrm{a}$ & $13,9 \pm 9,74 a$ & $17,4 \pm 8,32 \mathrm{a}$ & $11,5 \pm 6,46 \mathrm{a}$ & 6,32 & 38,6 \\
\hline Diabrotica speciosa & $0,4 \pm 0,04 \mathrm{a}$ & $0,6 \pm 0,05 a$ & $0,6 \pm 0,03 \mathrm{a}$ & $0,4 \pm 0,11 \mathrm{a}$ & 0,46 & 81,1 \\
\hline Maecolaspis assimilis & $0,2 \pm 0,14 \mathrm{a}$ & $0,2 \pm 0,26 a$ & $0,1 \pm 1,22 \mathrm{a}$ & $0,3 \pm 1,78 a$ & 0,20 & 91,9 \\
\hline Maecolaspis sp. & $4,1 \pm 2,45 \mathrm{a}$ & $3,3 \pm 1,69 a$ & $2,4 \pm 1,65 b$ & $5,0 \pm 1,88 a$ & 2,38 & 57,2 \\
\hline Neomegalotomus sp. & $0,7 \pm 0,29 a$ & $0,5 \pm 0,62 \mathrm{a}$ & $0,8 \pm 0,03 \mathrm{a}$ & $0,4 \pm 0,02 a$ & 0,38 & 59,1 \\
\hline Megascelis sp. & $10,4 \pm 2,63 a$ & $14,5 \pm 8,82 \mathrm{a}$ & $7,9 \pm 3,10 b$ & $17,0 \pm 7,34 \mathrm{a}$ & 4,65 & 33,1 \\
\hline Piezodorus guildinii & $1,0 \pm 0,45 a$ & $0,8 \pm 0,20 a$ & $0,8 \pm 0,70 a$ & $1,0 \pm 0,97 a$ & 0,50 & 49,3 \\
\hline Cycloneda sanguinea & $2,4 \pm 0,19 a$ & $1,0 \pm 0,32 b$ & $1,1 \pm 0,18 b$ & $2,3 \pm 0,26 a$ & 0,58 & 30,0 \\
\hline Doru sp. & $0,4 \pm 0,03 a$ & $0,7 \pm 0,01 \mathrm{a}$ & $0,2 \pm 0,01 b$ & $0,8 \pm 0,30 a$ & 0,51 & 89,1 \\
\hline Geocoris sp. & $0,8 \pm 0,02 \mathrm{a}$ & $0,9 \pm 0,30 a$ & $0,3 \pm 0,03 b$ & $1,5 \pm 0,28 a$ & 0,65 & 57,3 \\
\hline Toxom erus sp. & $0,8 \pm 0,18 b$ & $1,3 \pm 0,10 a$ & $0,6 \pm 0,03 b$ & $1,4 \pm 0,09 a$ & 0,44 & 38,8 \\
\hline
\end{tabular}

IInterações entre manejo do solo e culturas não foram significativas.

${ }^{2}$ Médias seguidas da mesma letra na horizontal não diferem pelo teste de Tukey a $5 \%$.

inimigos naturais (Letourneau, 1987). O milho pode ter contribuído para aumentar a abundância de insetos predadores na soja devido afetarem a turbulência do vento nas faixas de soja. Quebra-ventos são conhecidos por aumentarem a concentração de inimigos naturais em soja (Mayse \& Price, 1978). A presença do milho pode ter proporcionado alimento para insetos predadores. Joaninhas da espécie Coleomegilla maculata foram observadas alimentando-se de pólen de milho depositado em folhas de soja (Risch et al., 1982).

\section{AGRADECIMENTOS}

À FAPESP, pelo suporte financeiro para a realização deste trabalho. Aos Drs. José Carlos Barbosa, FCAV-UNESP, e Carlos Campaner, Museu de ZoologiaUSP, pelo auxílio nas análises estatísticas e pela identificação de várias espécies de insetos citadas neste trabalho, respectivamente.

\section{REFERÊNCIAS BIBLIOGRÁFICAS}

ALTIERI, M.A. Agroecologia: as bases científicas da agricultura alternativa. Rio de Janeiro: PTA/FASE, 1989. 240p.

ALTIERI, M.A.; GLASER, D.L.; SCHMIDT, L.L. Diversification of agroecosystems for insect pest regulation: experiments with collards. In: GLIESSMAN, S.R. (Ed.) Agroecology, researching the ecological basis for sustainable agriculture. New York: Springer-Verlag, 1990. p.70-82.

BECHINSKI, E.J.; PEDIGO, L.P. Ecology of predaceous arthropods in lowa soybean agroecosystems. Environmental Entomology, v.10, p.771-778, 1981.

BEST, L.R.; BEEGLE, C.C. Food preferences of five species of carabids commonly found in lowa cornfields. Environmental Entomology, v.6, p.9$12,1977$.

BIANCO, R. Ocorrência de pragas no plantio direto e convencional. In: FANCELLI, A.L.; TORRADO, P.V.; MACHADO, J. (Coord.) Atualização em plantio direto. Campinas: Fundação Cargill, 1984. p.183-194.

BOOIJ, C.J.H.; NOORLANDER, J. Farming systems and insect predators. Agriculture, Ecosystems and Environment, v.40, p.125-135, 1992.

BRUCK, D.J.; LEWIS, L.C. Influence of adjacent cornfield habitat, trap location, and trap height on capture numbers of predators and a parasitoid of the European corn borer (Lepidoptera: Pyralidae) in central lowa. Environmental Entomology, v.27, p.1557-1562, 1998.

BRUST, G.E.; STINNER, B.R.; MACARTNEY, D.A. Predation by soil inhabiting arthropods in intercropped and monoculture agroecosystems. Agriculture, Ecosystem and Environment, v.18, p.145-154, 1986. 
CÁRCAMO, H.A.; SPENCE, J.R. Crop type effects on the activity and distribution of ground beetle (Coleoptera:Carabidae). Environmental Entomology, v.23, p.684-692, 1994.

CASTRO, M.T.; PITRE, H.N.; MECKENSTOCK, D.H. Fall armyworm and neotropical cornstalk borer on sorghum and maize intercropped with legumes in Honduras. Turrialba, v.44, p.77-86, 1994.

CLARK, M.S.; GAGE, S.H.; SPENCE, J.R. Habitats and management associated with common ground beetles (Coleoptera: Carabidae) in a Michigan agricultural landscape. Environmental Entomology, v.26, p.519527, 1997.

COLL, M.; BOTTRELL, D.G. Microhabitat and resource selection of the European corn borer (Lepidoptera: Pyralidae) and its natural enemies in Maryland field corn. Environmental Entomology, v.20, p.526-533, 1991.

COTTRELL, T.E.; YEARGAN, K.V. Effect of pollen on Coleomegilla maculata (Coleoptera: Coccinellidae) population density, predation, and cannibalism in sweet corn. Environmental Entomology, v.27, p.1402-1410, 1998.

CRUZ, I.; OLIVEIRA, A.C. Flutuação populacional do predador Doru luteipes Scudder em plantas de milho. Pesquisa Agropecuária Brasileira, v.32, p.363-368, 1997.

ELLSBURY, M.M.; EXNER, D.N.; CRUSE, R.M. Movement of corn rootworm larvae (Coleoptera: Chrysomelidae) between border rows of soybean and corn in a strip intercropping system. Journal of Economic Entomology, v.92, p.207-214, 1999

FERGUSON, H.J.; MCPHERSON, R.M.; ALLEN, W.A. Effect of four soybean cropping systems on the abundance of foliage-inhabiting insect predators. Environmental Entomology, v.13, p.1105-1112, 1984.

FORNASIERI FILHO, D.A. Cultura do milho. Jaboticabal: FUNEP, 1992. 273p.

FRANK, J.H. Carabidae (Coleoptera) as predators of the readacked cutworm (Lepidoptera: Noctuidae) in central Alberta. Canadian Entomologist, v.103, p.1039-1044, 1971.

FRENCH, B.W.; ELLIOTT, N.C.; BERBERET, R.C. Reverting conservation reserve program lands to wheat and livestock production: effects on ground beetles (Coleoptera: Carabidae) assemblages. Environmental Entomology, v.27, p.1323-1335, 1998.

FULLER, B.W. Predation by Calleida decora (F.) (Coleoptera: Carabidae) on velvetbean caterpillar (Lepidoptera: Noctuidae) in soybean. Journal of Economic Entomology, v.81, p.127-129, 1988.

HAMMOND, R.B.; STINNER, B.R. Soybean foliage insects in conservation tillage systems: Effect of tillage, previous cropping history, and soil insecticide application. Environmental Entomology, v.16, p.524-531, 1987.

HSIN, C.Y; SELLERS, L.G.; DAHM, P.A. Seasonal activity of carabids and the toxicity of carbofuran and terbofos to Pterostichus chalcites. Environmental Entomology, v.8, p.154-159, 1979.

LETOURNEAU, D.K. The enemies hypothesis: tritrophic interactions and vegetational diversity in tropical agroecosystems. Ecology, v.68, p.1616$1622,1987$.
MAYSE, M.A.; PRICE, P.W. Seasonal development of soybean arthropod communities in East Central Illinois. Agro-Ecosystems, v.4, p.387-405, 1978

MILANEZ, J.M. Ocorrência de insetos em culturas consorciadas e monoculturas. In: ENCONTRO NACIONAL DE FITOSSANITARISTAS, 3. Florianópolis. Anais. 1984. Florianópolis, 1984. p.103-115.

MOREIRA, G.R.P.; BECKER, M. Mortalidade de Nezara viridula (Linnaeus, 1758) (Heteroptera: Pentatomidae) no estágio do ovo na cultura da soja. Anais da Sociedade Entomológica do Brasil, v.15, p.271-290, 1986.

PICANCCO, M.; LEITE, G.L.D.; MADEIRA, N.R.; SILVA, D.J.H. da; MIYAMOTO, A.N. Efeito do tutoramento do tomateiro e seu policultivo com milho no ataque de Scrobipalpuloides absoluta (Meyrick) e Helicoverpa zea (Bod.) Anais da Sociedade Entomológica do Brasil, v.25, p.175-180, 1996.

RAMASWAMY, S.B. Host finding by moths: sensory modalities and behaviours. Journal of Insect Physiology, v.34, p.235-249, 1988

RISCH, S.J.; WRUBEL, R.; ANDOW, D.F. Foraging by a predaceous beetle, Coleomegilla maculata (Coleoptera: Coccinellidae), in a polyculture: effects of plant density and diversity. Environmental Entomology, v.11, p.949950, 1982.

RISCH, S.J.; ANDOW, D.A.; ALTIERI, M.A. Agroecosystem diversity and pest control: data, tentative conclusions, and new research directions. Environmental Entomology, v.12, p.625-629, 1983

ROOT, E.P. Organization of a plant-arthropod association in simple and diverse habitats: the fauna of collards (Brassica oleracea). Ecologica Monograph, v.43, p.95-124, 1973.

SILVEIRA NETO, S.; NAKANO, O.; BARBIN, D.; VILLA NOVA, N. Manual de ecologia dos insetos. São Paulo: Ceres, 1976. 419p.

SOSA-GOMEZ, D.R.; MOSCARDI, F. Effect of till and no-till soybean cultivation on dynamics of entomopathogenic fungi in the soil. Florida Entomologist, v.77, p.93-95, 1994.

STINNER, B.R.; HOUSE, G.J. Arthropods and other invertebrates in conservation-tillage agriculture. Annual Review of Entomology, v. 35 , p.299-318, 1990.

STINNER, B.R.; McCARTNEY, D.A.; DOREN JR., D.M. van Soil and foliage arthropod communities in conventional, reduced and no-tillage corn (maize, Zea mays L.) systems: a comparison after 20 years of continuous cropping Soil and Tillage Research, v.11, p.147-158, 1988

TONHASCA JR., A. Effect of agroecosystem diversification on natural enemies of soybean herbivores. Entomologia Experimentalis et Applicata, v.69, p.83-90, 1993.

TONHASCA JR., A.; STINNER, B.R. Effects of strip intercropping and notillage on some pests and beneficial invertebrates of corn in Ohio. Environmental Entomology, v.20, p.1251-1258, 1991.

Recebido em 30.10 .00 